\title{
Crystal plasticity and HR-EBSD analysis of full-field polycrystal Ni superalloy strains and rotations under thermal loading
}

\author{
Tiantian Zhang ${ }^{1}$, David M. Collins ${ }^{2}$, Fionn P. E. Dunne ${ }^{1}$, Barbara A. Shollock ${ }^{1,3}$ \\ ${ }^{1}$ Department of Materials, Imperial College London, London SW7 2AZ, UK \\ ${ }^{2}$ Department of Materials, University of Oxford, Oxford OX1 3PH, UK \\ ${ }^{3}$ WMG, University of Warwick, Coventry CV4 7AL, UK
}

\begin{abstract}
Electron back scattered diffraction (EBSD) has been employed to study a polycrystalline nickel superalloy containing a complex non-metallic agglomerate under thermal loading. Heterogeneous distributions of elastic strains are observed near the inclusion due to its complex geometry which have been quantified. Lattice rotations were also related to geometrically necessary dislocation (GND) density $\left(\sim 10^{14} \mathrm{~m}^{-2}\right)$, indicating the development of localized plasticity arising from the mismatch in thermal expansivity between the Ni polycrystal and the inclusion. A crystal plasticity finite element (CPFE) model which explicitly represents the full detail of the complex microstructure was developed to interpret the experimental measurements, and good quantitative and qualitative agreement has been obtained. However, a limitation of the EBSD technique when investigating polycrystal systems is that full-field, trans-granular strain measurement remains difficult due to the necessity to reference a lattice spacing within a grain for strain calculation. An inverse reference shifting methodology has been developed using CPFE modelling to overcome this problem, thereby allowing like-for-like and grain-by-grain strain comparisons to be made. The method, in conjunction with high resolution-EBSD, shows promise for the determination of fullfield strains and rotations in polycrystalline materials, and provides key information for fatigue nucleation in these material systems.
\end{abstract}


Keywords: powder metallurgy nickel superalloys; residual strains; crystal plasticity; HR-EBSD

\section{Introduction}

Polycrystalline nickel based superalloys are uniquely capable of delivering exceptional mechanical properties when subjected to high temperatures within a corrosive environment, enabling their extensive use for gas turbine disc applications. The mechanical performance of these alloys arises from the presence of a multimodal distribution of coherent, $\mathrm{L1}_{2} \gamma^{\prime}$ (gamma-prime) precipitates, which exhibit a cube-cube orientation relationship with the A1 $\gamma$ (gamma) matrix. Due to the levels of segregation in conventional cast-wrought processing and the need to impose deformation straining, there is increasing interest in producing superalloy disk components via a powder metallurgy route. Despite the fact that great efforts have been introduced to improve powder cleanliness, contamination from refractories in contact with molten metal are inevitable and result in the formation of inclusions. Commonly observed inclusions in modern nickel superalloys include such non-metallic inclusions. These particles severely degrade the mechanical properties. In particular, the resistance to fatigue loading is strongly affected because cracks usually initiate from these inherent features and can rapidly propagate with the aid of loading, sometimes resulting in unpredictable failures [1-3].

Much work has been carried out to study the influence of inclusions on fatigue crack nucleation and propagation in nickel superalloys. Temperature [4, 5], grain size [6, 7], stress level [8], strain range [9], and size of non-metallic inclusions [10] have been found to affect fatigue performance. These microstructural features (grain boundaries, inclusions) introduce dislocation barriers and thus give rise to accumulation of damage, resulting in crack nucleation. However, if the effects of inclusions are to be well understood, a detailed study of elastic strain and stress accumulation as well as lattice distortion local to the heterogeneity must be conducted at appropriate length scales in conjunction with experimental validation. 
Cross-correlation based high resolution EBSD (HR-EBSD) has become an emerging tool for mapping elastic strains at mesoscopic and microscopic scales $[11,12]$ and reveals microstructural mechanisms of deformation. The geometrically necessary dislocation (GND) density can be measured by analysis of lattice curvature measured through Nye's tensor [13] and hence provides information on plastic deformation. To date, this technique has been used to study anisotropic behavior of hcp crystals [14], GND distributions after tensile deformation in Ti [15], macrozones in hot-rolled Ti-6Al-4V [16], residual stresses and GND accumulation in annealed [17, 18] and deformed copper [19], and transformation-induced GND development in steels [20]. In particular, a recent application of this technique to obtain strain and rotation fields around a carbide particle in a single crystal nickel matrix under thermal loading has shown both qualitative and quantitative agreement with gradient enhanced CPFE predictions [21, 22]. However, the quantitative determination of full-field, absolute elastic strain and lattice rotation distributions in polycrystalline metals using HR-EBSD is currently not available due to the so-called 'reference pattern' problem [23].

Crystal plasticity finite element analysis is a desirable tool to probe microscopic deformation. A significant driver is to develop a cost-effective way to predict accurately the evolving plasticity in industrially relevant engineering alloys under relevant loading conditions [24, 25]. Application of this technique is widespread and can be found in studies of anisotropy in Ti $[14,24,26]$, fatigue crack nucleation in steels [27], and thermal residual stresses in nickel superalloys [22]. A remaining challenge is to capture accurately the full details of microscopic strain, stress, lattice rotation, and GND density measured by experiments, and particularly with HR-EBSD [28]. The use of Bragg's law simulation patterns is a potential way to overcome this challenge [29], but the geometric configuration of EBSD camera and sample must be obtained with great accuracy. Common uncertainties in pattern centre lead to errors of about $10^{-3}$ in strain measurement [30]. However, the selection of the simulation method may have significant effects on shift measurement [29]. 
Therefore, the level of fidelity and uncertainty associated with pattern simulation motivates the development of alternative techniques to overcome the 'reference pattern' problem.

In this paper, a CPFE model and high resolution EBSD are employed to study a non-metallic inclusion in a powder metallurgy produced polycrystalline nickel based superalloy (RR1000). When subjected to stress relief by heat treatment at $1033 \mathrm{~K}$, significant strains develop due to differing thermal expansivities of the inclusion and nickel matrix. Here, we present elastic strains and lattice rotations determined by both model and experiment. Qualitative and quantitative comparisons are then performed. A reference shifting methodology is introduced to reference correctly the strain and rotation fields, hence enabling detailed, full-field comparisons between the HR-EBSD-measured results and those predicted using CPFE. Correctly referencing polycrystal strains and rotations in HR-EBSD is termed direction correction, but currently, direct experimental methodologies to do this are not available. Hence, in this paper, inverse 'referencing' of strains and rotations from the CPFE model is performed in order to enable detailed, full-field polycrystal comparisons between experimental measurements and model predictions.

\section{Materials and methodology}

RR1000 samples were supplied by Rolls-Royce plc and were cut down to a dimension of $6 \times 5 \mu \mathrm{m}$ for microscopic characterization. The nominal composition of the alloy is given in Table 1 . The sample was produced via a powder metallurgy route followed by extrusion, forging and a two-step heat treatment. A sub-solvus solution heat treatment was conducted at $1393 \mathrm{~K}$ for four hours and then forced air quenched. Upon cooling, the $\gamma$ matrix becomes sufficiently supersaturated that a fine dispersion of intragranular $\gamma^{\prime}$ precipitates homogenously nucleate and grow until the diffusion fields overlap, resulting in soft impingement [31]. The material was next subjected to an aging heat treatment at $1033 \mathrm{~K}$ for sixteen hours where the intragranular precipitates coarsen in a controlled manner to provide precipitate size distributions which are tailored to yield the best overall 
performance of a turbine disk component. Notably, the resulting fine grain microstructure, combined with the multi-modal distribution of $\gamma^{\prime}$ precipitates, results in good low cycle fatigue properties.

\begin{tabular}{|l|l|l|l|l|l|l|l|l|l|l|l|}
\hline element & Ni & Co & Cr & Mo & Ta & Ti & Al & B & C & Zr & Hf \\
\hline wt\% & bal. & 18.5 & 15 & 5 & 2 & 3.6 & 3 & 0.015 & 0.027 & 0.06 & 0.5 \\
\hline
\end{tabular}

Table 1. Nominal composition of the nickel-base superalloy, RR1000 [32].

A non-metallic inclusion was detected from an evaluation of microstructure in the RR1000 sample, which was then carefully sectioned to leave the inclusion on the surface. Standard metallographic grinding and polishing were performed to achieve a suitable surface quality for scanning electron microscopy (SEM) and Energy Dispersive X-ray (EDX), after which a final light polish with colloidal silica for ten minutes was applied to obtain the optimum surface finish required for subsequent EBSD analysis.

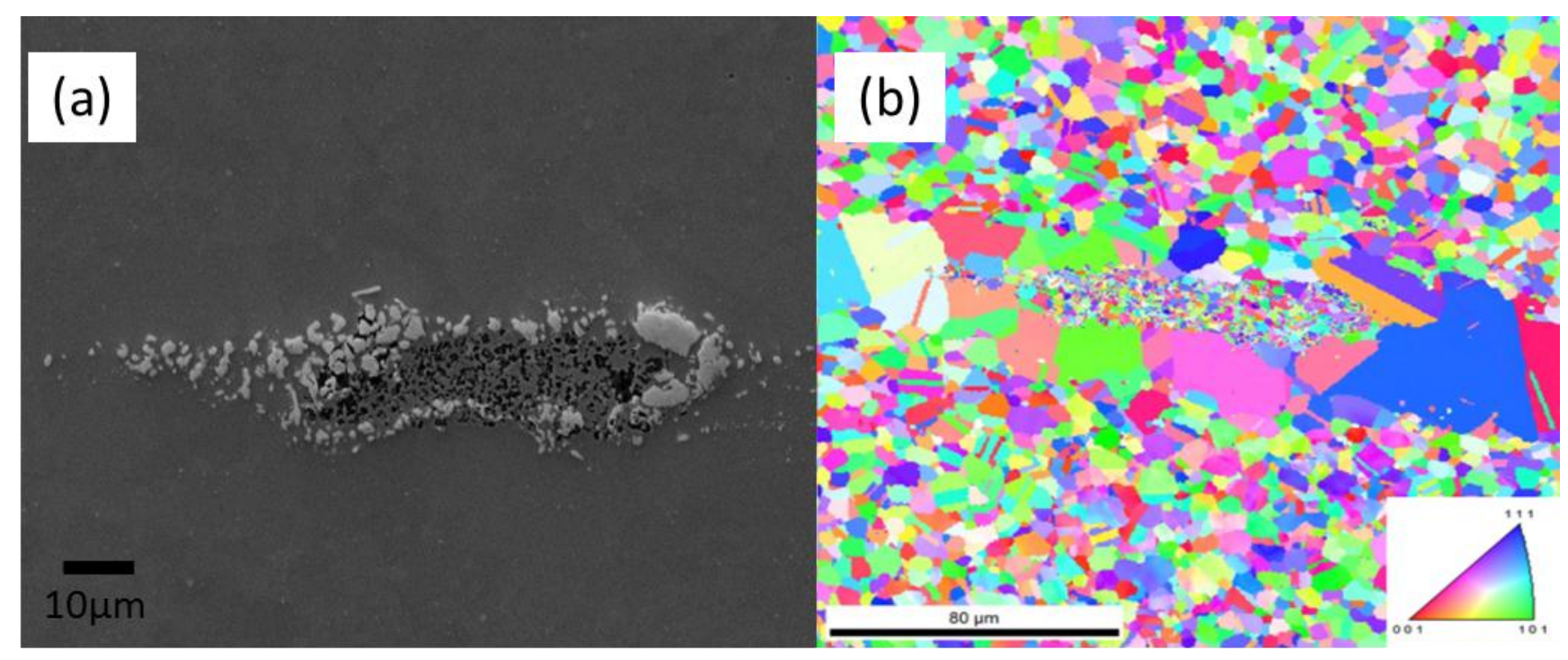

Figure 1. (a) BSE image of the inclusion; (b) EBSD map with the corresponding inverse pole figure showing crystallographic orientations 
A back-scattered electron (BSE) image from the inclusion was obtained on a Carl Zeiss Auriga scanning electron microscope operating at an accelerating voltage of $20 \mathrm{kV}$ and a beam current of 10 nA. An agglomerate of particles with a distinct two-phase contrast is observed as seen in Fig. 1 (a), indicating a significant compositional difference. Elements present in the outer rim of the agglomerate have higher atomic number than those in the interior. The inclusion is elongated in one direction and it has a dimension of approximately $110 \times 20 \mu \mathrm{m}$.

EDX measurements were performed using Inca from Oxford Instruments on a JEOL 6300 tungsten filament scanning electron microscope. The chemical analysis demonstrates that the inclusion includes hafnium oxide particles surrounded by particles of aluminum oxide with nickel alloy islands in between. Previous investigations [4, 33-35] have suggested that such an inclusion is formed during the manufacturing process as a result of reactions between melting crucible and alloying elements.
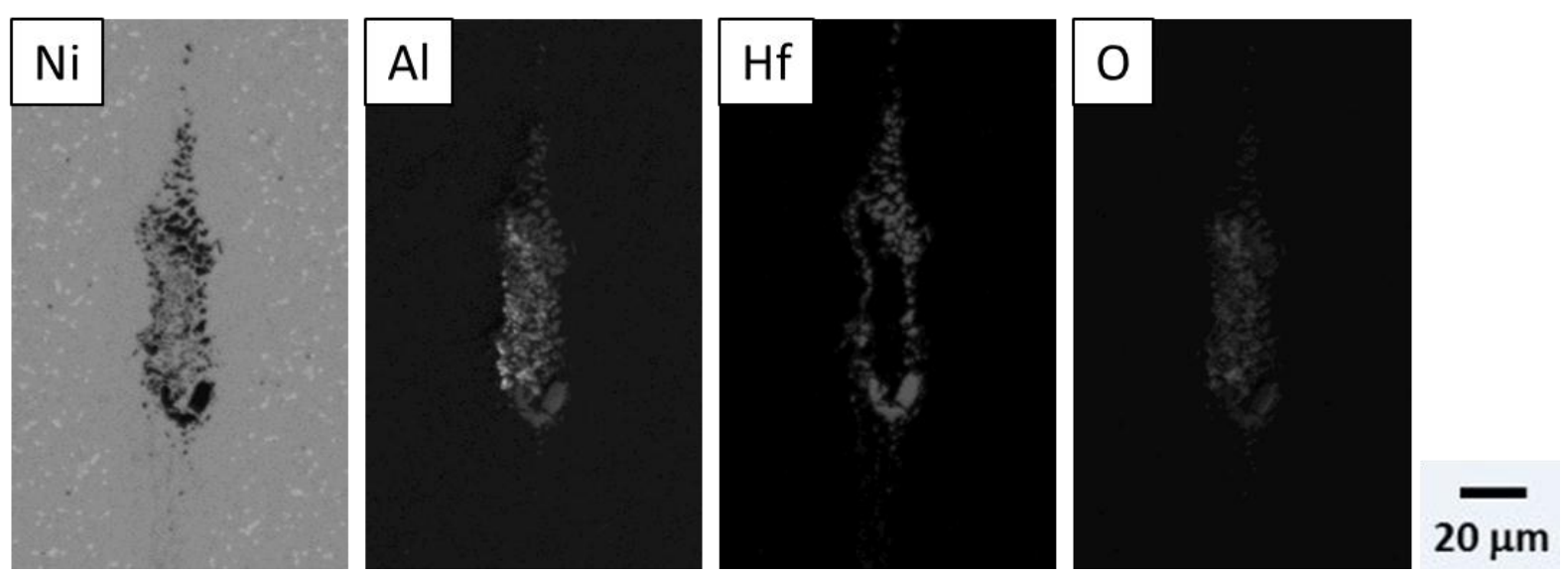

Figure 2. Energy dispersive X-ray maps of selected alloying elements in inclusion presented in Fig.

1. Note that the intensities were adjusted to improve the contrast and therefore cannot be used to make quantitative comparisons. Light grayscale values correspond to higher element content. 
A JEOL 6500F (FEG) scanning electron microscope was used at an accelerating voltage of 20kV and a probe current of approximately $10 \mathrm{nA}$, to conduct EBSD characterization to obtain the crystallographic orientations of the grains in close proximity to the inclusion. Diffraction patterns were collected using a TSL Digiview II camera with an acquisition time of $\sim 1$ second, operating with $1 \times 1$ binning and a $0.45 \mu \mathrm{m}$ step size which is significantly larger than the spatial resolution of the EBSD camera. The step size was chosen to balance conflicting factors, such as Burger's circuit size, angular resolution, scanning time, sufficient number of data points within each grain. The data was collected using the imaging microscopy (OIM) DC v6 software, in which the orientations are described by the three Euler angles in Bunge notation. Preliminary EBSD characterization, as shown in Fig. 1(b), revealed that the surrounding grains exhibit a random distribution of orientations.

\subsection{High resolution EBSD}

After the aging step, significant strains developed at the particle/metallic matrix interface due to differences in the expansivities of the nickel crystal and the oxide phase. The elastic strains were measured by means of high resolution EBSD from the deformation causing shifts in zone axes in the diffraction patterns. An area of $201.45 \times 206.10 \mu \mathrm{m}$ containing the inclusion was selected within which a cropped $1000 \times 1000$ pixel EBSD pattern was obtained at each interrogation point. The results were digitized to 12 bits and stored in a hard disk for off-line analysis. Shifts at 20 regions of interest (ROI) separately located across a diffraction pattern were measured using an automatic image processing technique based on cross-correlation analysis in order to calculate eight of the nine degrees of freedom in the deformation tensor. The last degree of freedom, which is the hydrostatic dilation, was determined by assuming zero traction since the interaction volume is typically approximately $20 \mathrm{~nm}$. The fully resolved tensor was then used to calculate the elastic strains and lattice rotations $[11,36]$. 'Traditional EBSD' performed in the Hough space has a noise level of $10^{-3} \mathrm{rad}$ for misorientation measurement. In comparison, image cross correlation analysis of 
regions of interest in diffraction patterns, performed in Fourier domain via a 2-D fast Fourier transform, allows lattice misorientation measurement to achieve a sensitivity of about $10^{-4} \mathrm{rad}$ without sacrificing spatial resolution [11]. Therefore, HR EBSD with higher angular resolution is capable of capturing local GND distributions whereas Hough-based EBSD is only sensitive to coarser dislocation structures [37]. For each grain, the location with the highest image quality was selected as a reference and elastic strains and rotations were determined relative to the reference.

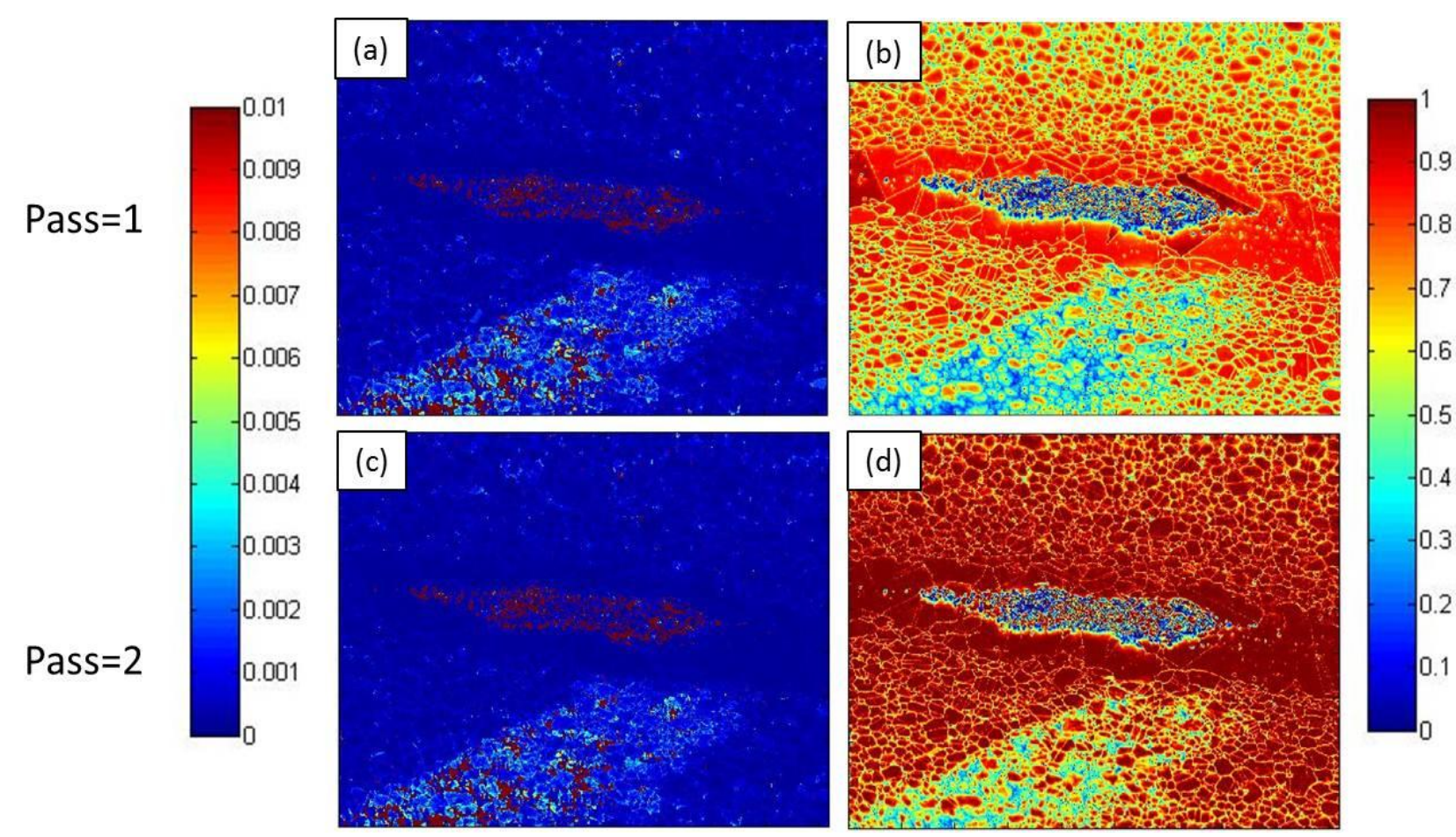

Figure 3. Mean angular error (a and c), peak height ( $b$ and d) of the EBSD analysis after pass 1 and

The mismatch of thermal expansivity is large enough to result in plasticity $[21,22]$ and hence large gradients in lattice rotation (above $4^{\circ}$ ) may be expected at the particle/matrix interface. This can make initial upsampling of cross-correlation peak inaccurate, resulting in problems in strain measurement [38]. To rectify this, a first pass of cross-correlations was applied to calculate the rotation components required to remap the test diffraction pattern back to the orientation of the reference. Remapping of intensities in the test pattern was undertaken to make sure that only elastic 
strains and small differences in lattice orientation were considered. ROIs were then selected from the remapped test pattern and were cross-correlated to those in the reference to measure the shifts. Finally, strains were determined with enhanced accuracy by combining the rotation correction for remapping with the small lattice rotation and elastic strains measured in the second pass.

Fig. 3 illustrates the importance of pattern remapping where both mean angular error and peak height after first and second pass are shown. Cross-correlation pattern matching can be assessed by calculating the peak height where transformed peaks of ROIs on test and reference patterns are compared and normalized to 1 for auto-correlation. Mean angular error is descriptive of the level of consistency of the shifts predicted by a (robust) best-fit solution and those actually measured. Performing the remapping has reduced the mean angular error and increased peak height around the inclusion. This, in return, makes the corresponding measurements more reliable.

\subsection{Crystal plasticity finite element implementation}

A brief description of the development of the crystal plasticity model for this analysis is given below. A more detailed illustration of the model and implementation of the physically-based constitutive equation using a finite element approach can be found in [24].

Flow kinematics describes a process where a lattice is deformed from an original configuration to the current configuration under external loading. The deformation gradient, $\boldsymbol{F}$, which maps the positions of a material point in the deformed state and the original state, can be decomposed multiplicatively into elastic and plastic parts [39]

$$
\boldsymbol{F}=\boldsymbol{F}_{e} \boldsymbol{F}_{P}
$$

The lattice configuration resulting from plastic deformation is formed by crystallographic slip. Therefore, it develops as 


$$
\dot{F}_{P}=L_{P} F_{P}
$$

where the plastic velocity gradient, $\boldsymbol{L}_{\boldsymbol{P}}$, embodies the dislocation slip rate, $\dot{\gamma}^{\boldsymbol{i}}$, on all the active slip (from i to n) system with slip direction, $\boldsymbol{s}$, and normal to the plane, $\boldsymbol{n}$, as

$$
\boldsymbol{L}_{\boldsymbol{P}}=\sum_{i=1}^{n} \dot{\gamma}^{i}(\boldsymbol{s} \otimes \boldsymbol{n})
$$

The formulation of the slip rate requires physical interpretation of the dynamics of lattice defects, such as dislocations, that act as elementary carriers for plastic flow. Dunne et al. [24] has developed a physically-based constitutive model that characterizes plastic slip rate on an active slip plane. The model originates from the slip rate of a single dislocation and considers the probability of dislocation jump over barriers due to thermal activation. For plasticity to occur, the resolved shear stress, $\tau$, must exceed the critical resolved shear stress, $\tau_{c}$. When plastic flow occurs, the slip rate becomes

$$
\dot{\gamma}=\rho b^{2} v \exp \left(-\frac{\Delta H}{k T}\right) \sinh \left(\frac{\left(\tau-\tau_{c}\right) \gamma_{0} \Delta V}{k T}\right)
$$

in which $\rho$ is the density of gliding dislocations, b Burger's vector, $v$ the successful frequency of a dislocation attempting to overcome a barrier, $\Delta \mathrm{H}$ Helmholtz free energy, $\mathrm{k}$ the Boltzman constant, $\mathrm{T}$ the temperature in Kelvin, $\gamma_{0}$ the work conjugate to the resolved shear stress at reference state, and $\Delta \mathrm{V}$ the activation volume. Length scale dependence is incorporated by the coupling of the density of GNDs with the slip rule where $\Delta V \propto \lambda_{P} b^{2}$ and $\lambda_{P} \propto \frac{1}{\sqrt{\rho_{G N D}+\rho_{S S D}}}$ and, where $\rho_{S S D}$ is the density of sessile statistically-stored dislocations (SSD), $\rho_{G N D}$ the density of GNDs, and $\lambda_{P}$ is the distance between the resulting pinning points (e.g., second phase precipitates, but here, mean free dislocation spacing). 

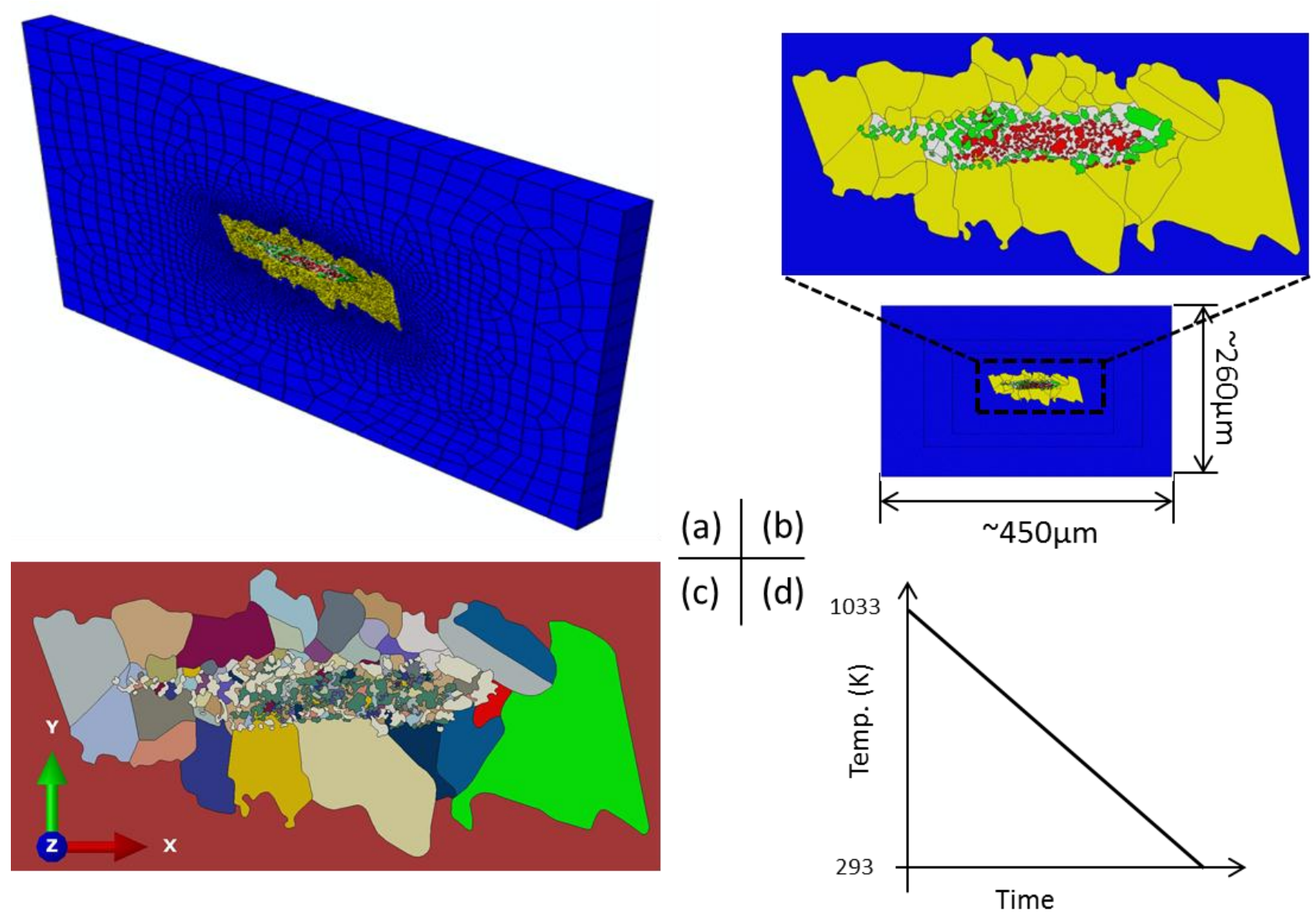

Figure 4. (a) Three-dimensional finite element model of the inclusion/matrix combination (b) front view of the model with a magnified view of the surrounding large grains with approximate dimension (c) random crystallographic orientations of the surrounding grains indicated by color and (d) the applied thermal loading

\begin{tabular}{|l|l|l|l|}
\hline Phase & $\begin{array}{l}\text { Young's Modulus } \\
(\mathrm{GPa})\end{array}$ & $\begin{array}{l}\text { thermal expansivity } \\
\left(10^{-6}{ }^{\circ} \mathrm{C}^{-1}\right)\end{array}$ & Poisson ratio \\
\hline Surrounding Ni grains & 207 & 13 & 0.28 \\
(yellow) & & & \\
\hline Ni grains away from the & RR1000 temperature-dependent properties from Rolls-Royce plc. \\
\hline
\end{tabular}




\begin{tabular}{|l|r|r|r|}
\hline inclusion (blue) & 207 & 13 & 0.28 \\
\hline small Ni grains in & 250 & 4.4 & 0.25 \\
between the oxide & & & \\
particles (white) & & & \\
\hline Hf-rich oxide (green) & 380 & 6.6 & \\
\hline Al-rich oxide (red) [41] & & & \\
\hline
\end{tabular}

Table 2. Material properties for the crystal plasticity model. Colour corresponds to the phase shown in Fig. 4 (b)

A geometric finite element representation of the inclusion/matrix combination, as shown in Fig. 4 (a), was developed using the commercial finite element package, ABAQUS. The model is meshed with 16,692 20-noded 3D quadratic User Defined Elements (UEL) with reduced integration. Plane stress conditions exist on the free surface. The bottom, left, and back faces are fixed in the $y, x$, and $\mathrm{z}$ directions, respectively, but are free to move in other directions. The conditions reproduce the assumptions made in the high resolution EBSD measurements that the out-of-plane normal stress is zero. Due to the absence of information on the $3 \mathrm{D}$ characterization and geometries of the agglomerate, the front face is assumed to be prismatic in the $\mathrm{z}$ direction. Appropriate sets of material properties (Table 2) are assigned to the Al-rich region, the Hf-rich region, the surrounding large grains, the grains in between the oxide particles, and the fine grains distant from the inclusion. The oxides are assumed to deform elastically because the resistance to dislocation motion is high due to their intrinsic lattice resistance. Considering that it does not have a preferred orientation and is distant from the oxides, elastic isotropy was used for the fine-grained region. Elastic anisotropic crystal plasticity is used for the large surrounding grains where crystallographic orientation plays an important role in local strain and stress fields and has been specified with knowledge of the 
orientations measured using EBSD (Fig. 4(c)). The orientations of the small grains in between the oxide particles are not accessible due to their relatively small size and therefore are assumed random by inspection of Fig. 1 (b). Thermal loading from $1033 \mathrm{~K}$ to room temperature is applied to reproduce the cooling process imposed on the sample (Fig. 4(d)).

\subsection{Referencing and shifting of elastic strain and lattice rotation}

For HR-EBSD strain measurement, cross-correlation functions are used to determine pattern shifts between test and reference patterns. It is assumed that the reference has zero strain or known strain state. Elastic strains and lattice rotations can be related to the shifts and subsequently calculated through the fully resolved deformation tensor. However, in many circumstances, a reference pattern with zero or known states cannot be obtained and so the resulting elastic strain and rotation measurements are relative, representative of the variations from the unknown elastic strain state at the reference. This causes problems for full-field strain measurements in polycrystalline materials, in which the EBSD-measured elastic strains are therefore given with respect to a reference strain state which is different in each grain. A consequence is that the elastic strain distributions so obtained within a given grain are in fact the variations of elastic strain from the reference point elastic strain in that grain. Further, the strain magnitudes cannot be compared transgranularly since each grain has its own reference point.

A potential approach to overcome this so-called 'reference pattern' problem and to enable likewith-like comparisons to be made between the model predictions and the EBSD measurements is to identify a grain-unique reference point within each grain, for which the CPFE model is able to provide the calculated elastic strain state. The EBSD-measured elastic strains may then be shifted to ensure equality of elastic strain with the CPFE at the grain reference point for each grain in turn. The methodology, therefore, in summary, is to use the CPFE elastic strain results in order to provide the reference strain for each grain to be used in the EBSD analysis. This then enables full- 
field, polycrystal elastic strain distributions providing like-with-like comparisons. This is possible because of the basic requirement of linearity in elasticity, since the EBSD technique explicitly measures elastic strains. The methodology is detailed schematically in Fig. 5 in which EBSD provides the elastic strain differences with respect to a reference point chosen within each grain at which zero elastic strain is assumed. A schematic example is shown in Fig. 5 (b). At this stage, the choice of the reference point from which strain variations are measured is arbitrary but is often chosen to be the point in the grain which has the best image quality. However, the crystal plasticity model, subject to the limitations of the underlying assumptions, enables full field calculations, and with knowledge of the reference point $\mathrm{P}$ chosen in Fig. 5 (c), the calculated elastic strains at this point are obtained from the CPFE, hence enabling the EBSD-measured strains within the grain to be shifted (by $\left.\Delta \varepsilon^{0}\right)$ to ensure that the EBSD-measured $\left(\varepsilon^{e^{\prime}}\right)$ and CPFE-calculated $\left(\varepsilon^{\mathrm{e}}\right)$ elastic strains at the reference point $\mathrm{P}$ in each grain are the same such that

$$
\varepsilon^{e^{\prime}}=\varepsilon^{\mathrm{e}}+\Delta \varepsilon^{0}
$$

In Appendix A, it is shown that the elastic strain shifting process is such that both the shifted and unshifted elastic strain, and stress distributions satisfy the requirements of compatibility, and pointwise and spatial equilibrium respectively. Indeed, it is shown that the grain-wise constant elastic strain shifts are simply equivalent to the incorporation of grain body forces, but which do not change the nature of the elastic strain fields. 


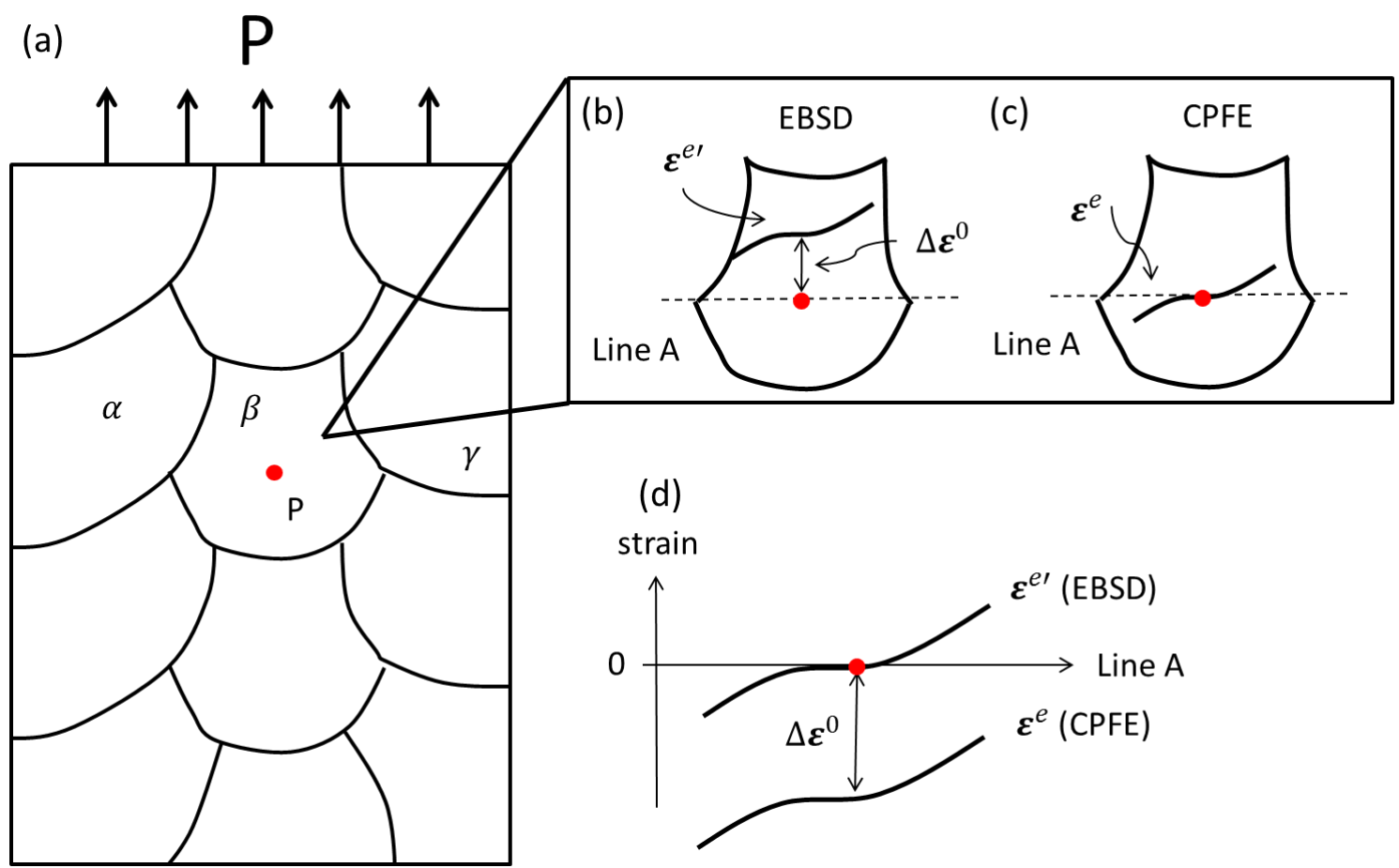

Figure 5. A 2-D polycrystal (a) with reference point $\mathrm{P}$ and for grain $\beta$ (b), the elastic strain difference $\left(\Delta \varepsilon^{0}\right)$ with respect to reference P measured by EBSD, (c) the CPFE-determined full-field elastic strains and (d) the elastic strain profile showing equality of elastic strain between $\mathrm{CPFE}$ and EBSD after shifting.

However, in this paper, in order to facilitate grain-by-grain comparison, the shifting is, instead, made to the CPFE-calculated results. First, the coordinates of the reference points selected for the EBSD referencing were obtained. The elastic strains at the corresponding locations in the CPFE model were then extracted and shifted such that all strains in a given grain were shifted relative to the same zero-strain reference points identified in the EBSD measurements. An example is shown in Fig. 6 where five large grains are selected and marked as 1-5 in Fig. 6(a). The EBSD-selected reference points are located and marked as black crosses in Fig. 6(b) and (c), and these points are also used in the CPFE post-processed results in order to ensure that at these reference points, the CPFE elastic strains are shifted to zero, and that all other elastic strains within the relevant grain are also shifted accordingly. The particular shifts applied to the grains studied are listed in Table 3 . The 
shifting of elastic strains and rotations is a linear operation which does not of itself alter the material or its state, and therefore does not introduce any additional errors in to the EBSD-measured strains and rotations.
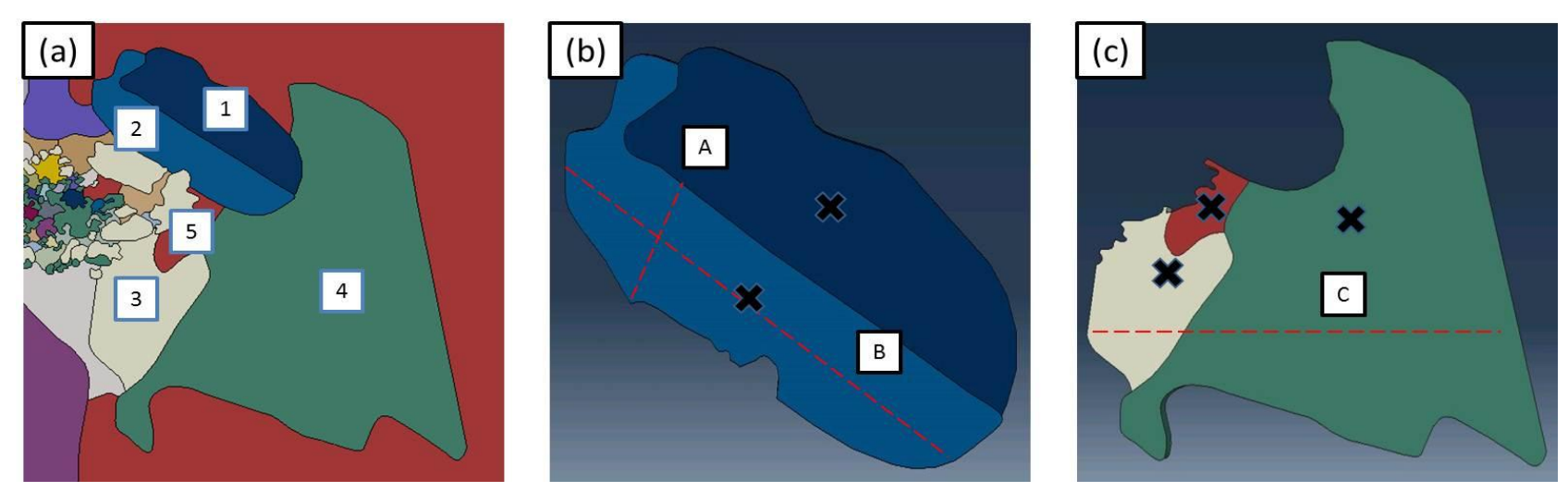

Figure 6. (a) Selected sets of grains for strain and lattice rotation referencing; (b) and (c) an enlarged view of the grain sets. The dashed lines are introduced for quantitative analysis (see later)

\begin{tabular}{|l|r|r|r|r|}
\hline Grain ID & \multicolumn{1}{|c|}{$\Delta \boldsymbol{\varepsilon}_{\boldsymbol{x} \boldsymbol{x}}^{\mathbf{0}}$} & \multicolumn{1}{|c|}{$\Delta \boldsymbol{\varepsilon}_{\boldsymbol{y} \boldsymbol{y}}^{\mathbf{0}}$} & \multicolumn{1}{c|}{$\Delta \boldsymbol{\varepsilon}_{\boldsymbol{x} \boldsymbol{y}}^{\mathbf{0}}$} & \multicolumn{1}{|c|}{$\Delta \boldsymbol{\omega}_{\boldsymbol{x} \boldsymbol{y}}^{\mathbf{0}}$} \\
\hline Grain 1 & 0.000357559 & -0.000471586 & -0.00130082 & -0.0007411 \\
\hline Grain 2 & -0.000161533 & 0.000522705 & -0.00254137 & -0.0019713 \\
\hline Grain 3 & 0.000300386 & -0.000469939 & 0.00100823 & 0.0014579 \\
\hline Grain 4 & -0.000520181 & 0.000351317 & 0.00039777 & 0.0000773 \\
\hline Grain 5 & 0.000591744 & -0.000384646 & 0.00335753 & 0.0013463 \\
\hline
\end{tabular}

Table 3. Magnitudes of elastic strains (column 2-4) and rotation (column 5) applied to the selected grains by the shifting method. 


\section{Results}
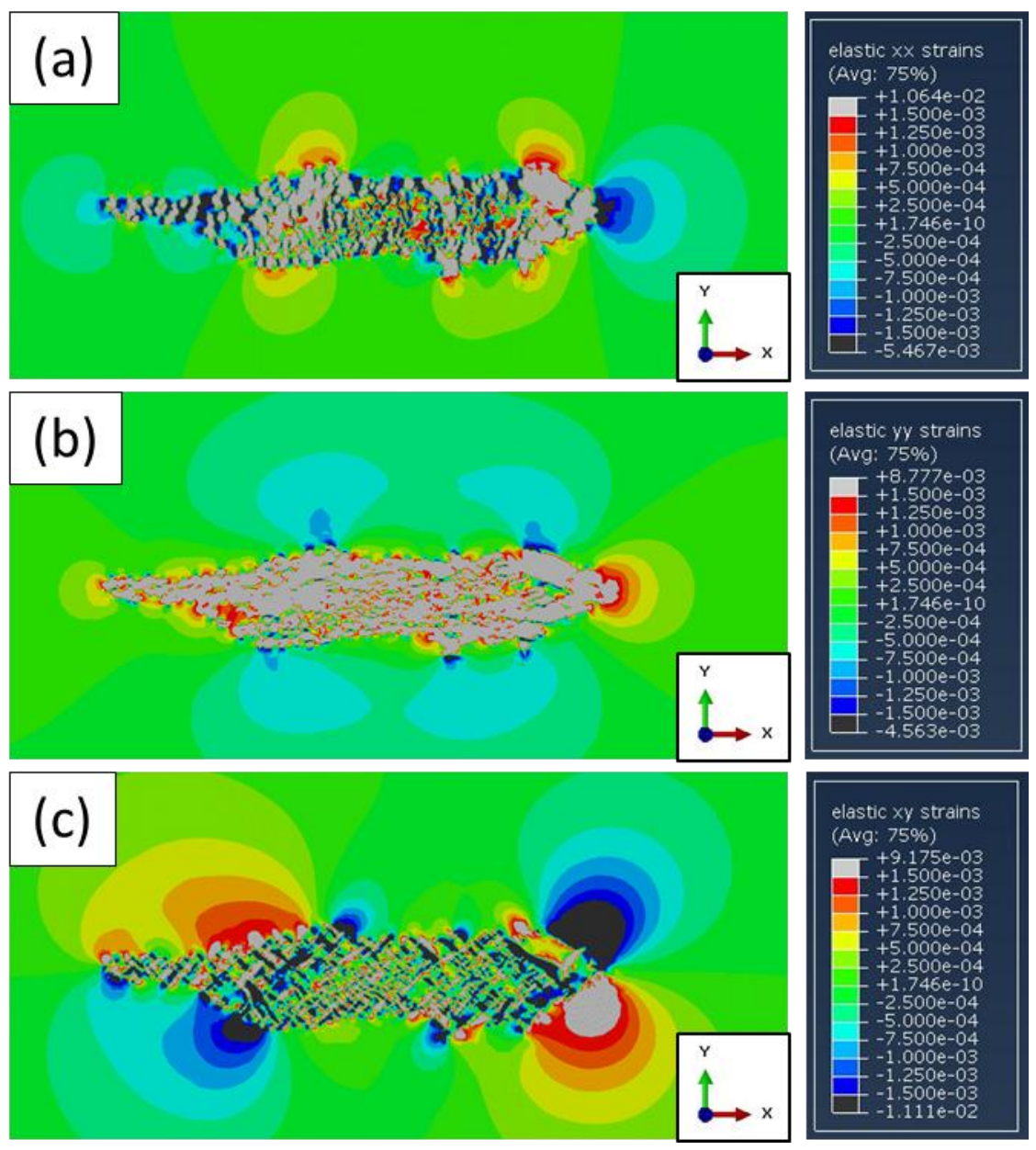

Figure 7. Field plots of CPFE predicted elastic strains in the xx (a), yy (b), and xy (c) directions.

The global, full-field results from the crystal plasticity analysis are considered first before more detailed, grain by grain comparisons with HR-EBSD measurements are considered. The model facilitates calculation of the elastic, thermal and plastic straining developed, but because the strains measurable using HR-EBSD are confined only to the elastic contributions (since thermal straining is dilatational), we limit in this section the discussion to elastic strains. It is noted that the average thermal expansivity of the surrounding nickel matrix is higher than that for the agglomerate such that the temperature change shown in Fig. 4(d) to which the agglomerate model is subjected is likely to lead to the following overall elastic strain behavior. For surfaces on the agglomeratematrix interface orientated approximately normal to the $\mathrm{x}$-axis, the $\mathrm{xx}$-strains are therefore 
anticipated to be compressive, and on surfaces arranged parallel to the $\mathrm{x}$-axis, the elastic strains are anticipated to be tensile. Conversely, for the yy strains, surfaces which are orientated approximately normal to the y-axis are expected to carry compressive strains, and those arranged about parallel to the y-axis should show tensile straining. The xy shear strains, on the other hand, are expected to be anti-symmetric. The elastic strains calculated from the crystal plasticity model are shown in Fig. 7 and may be seen to reproduce the overall anticipated characteristics just discussed.

The results of the EBSD-measured elastic strains in two sets of grains are shown in Fig. 8 and Fig. 9, together with the corresponding results predicted by the CPFE model. Strains before the reference-shifting are shown in the middle columns in Fig. 8 and 9 and those after referenceshifting are presented in the right-hand columns. Considerable disagreement can be seen between the experimentally measured and the CPFE results prior to the reference shifting. In particular, xy strains in Fig. 8 (c) and (f) can be seen to be opposite in sign. In contrast, quite considerable similarities are achieved after the correct reference shifting in grain 1 and 2. For instance, the reference-shifted strains in Fig. 8 (i) are approximately symmetric, being positive at the left and right ends and negative in the center, in a good agreement with the EBSD-measured strains (Fig. 8 (c)) in a qualitative sense. However, some features are clearly not captured by the reference-shifted CPFE model. Strain fields in Fig. 9 are apparently in less good agreement. However, this may be attributed to a surface scratch present close to these grains. 


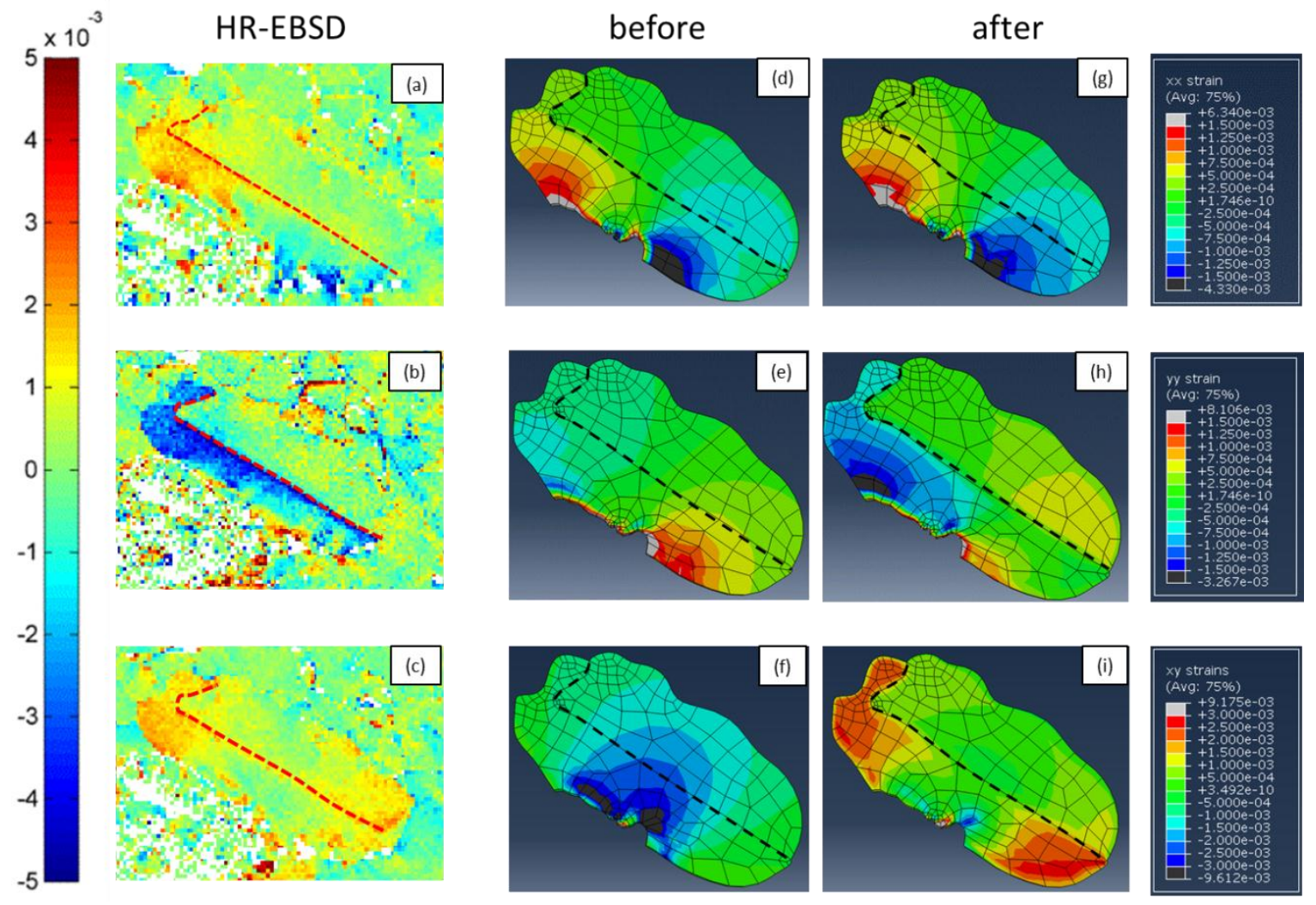

Figure 8. Field plots of EBSD determined (a-c) and CPFE predicted (d-i) elastic xx (first row), yy (second row), and xy strains (third row) in grain1 and 2. Pre-and post-reference-shifted results are shown in (d-f) and (g-i), respectively. The colour scale on the left relates to the experimental measurements and that on the right relate to the CPFE prediction, where both scales are provided since no one to one correspondence of colour exists. Grain boundaries are marked by the dashed lines. 

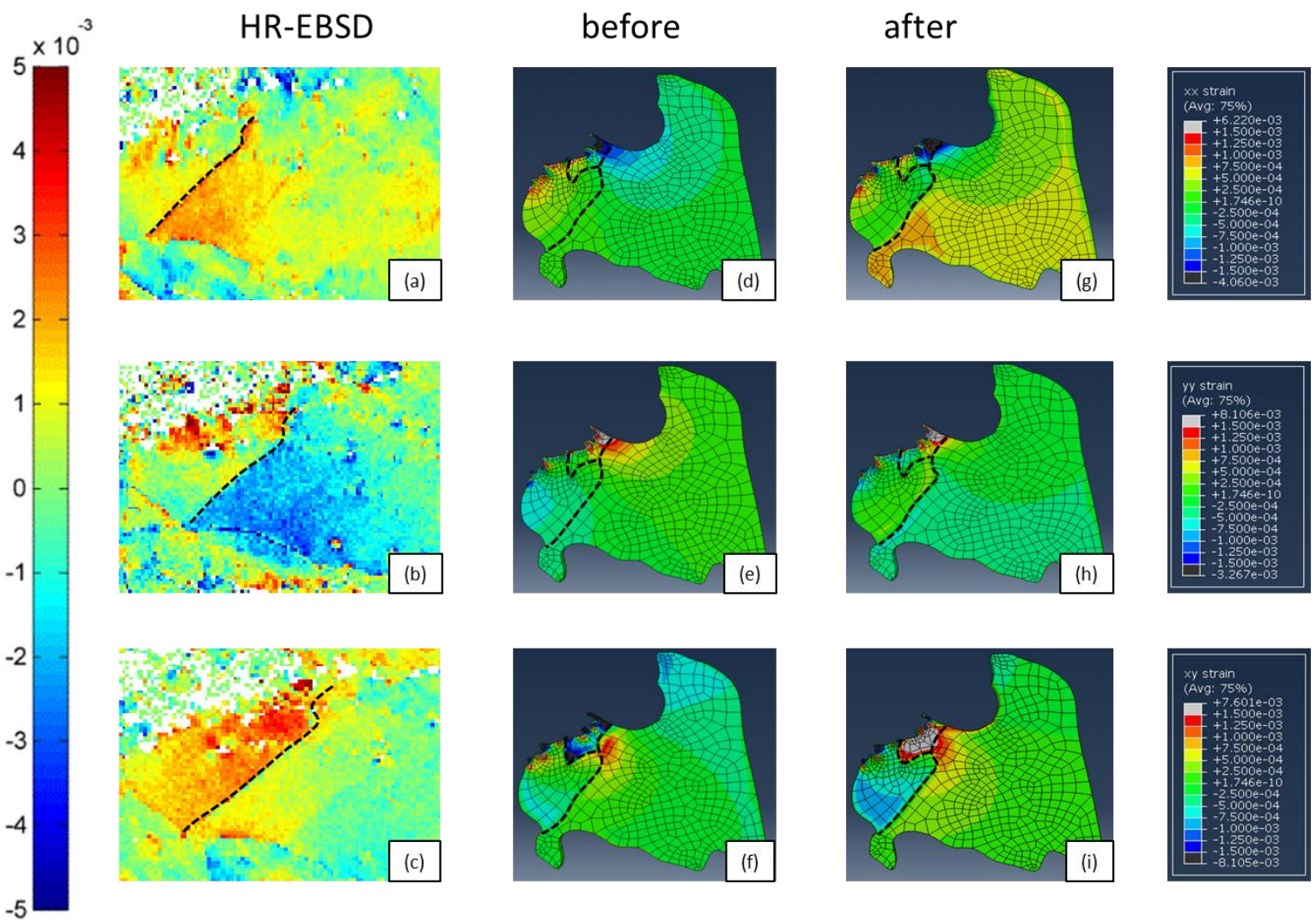

Figure 9. Field plots of EBSD (a-c) and CPFE predicted (d-i) determined elastic xx (first row), yy (second row), and xy strains (third row) in grain 3, 4, and 5. Pre-and post-reference-shifted results are shown in (d-f) and (g-i), respectively. The color scale on the left relates to the experimental measurements and that on the right relate to the CPFE prediction. Grain boundaries are marked by the dashed lines.

Due to the complex particle geometry, considerable variations in the accumulated plastic strains occur at the particle/nickel interface and gradients in plastic straining are therefore expected (see later). As a result, it is reasonable to anticipate lattice curvature and accumulation of geometrically necessary dislocations at the interface. Fig. 10 shows the EBSD determined and CPFE predicted inplane lattice rotations before and after the reference shifting. In both sets of grains (first and second rows), prior to reference shifting, the rotation fields have large differences relative to the EBSD measurements, but considerable agreement is achieved after the referencing. 
HR EBSD

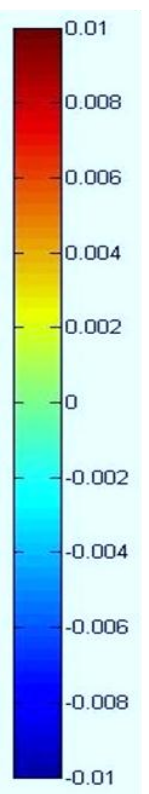

before
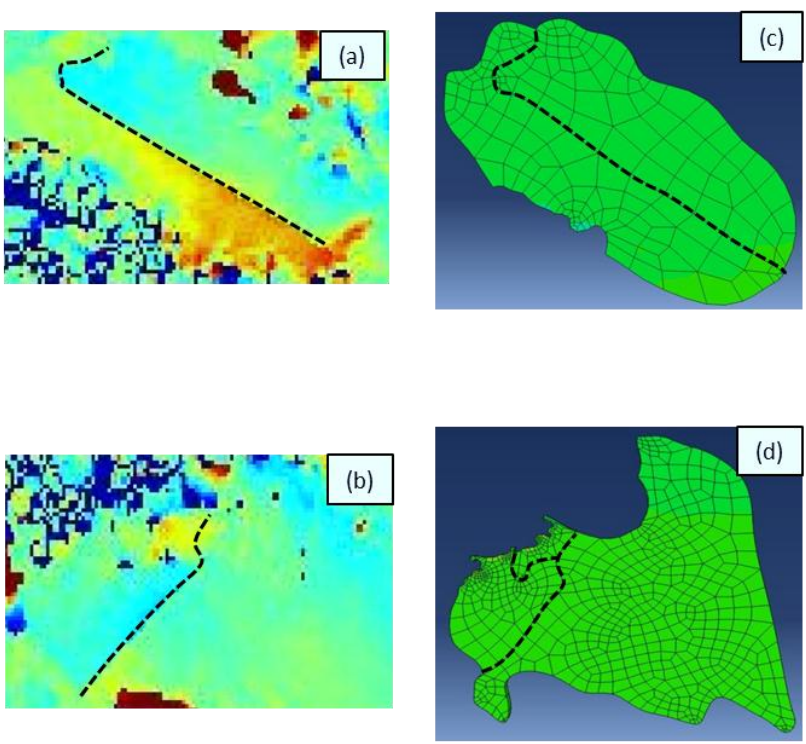

after
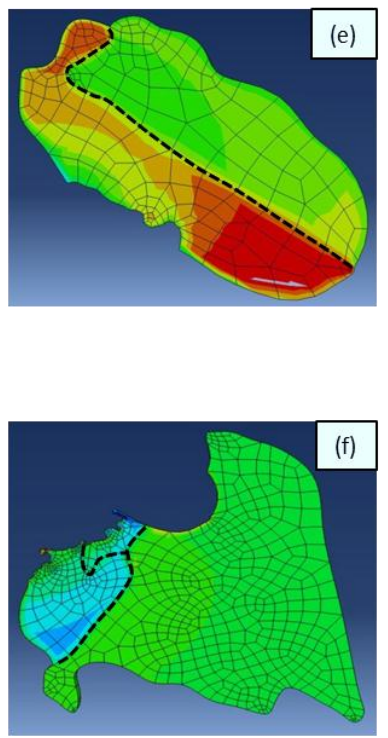

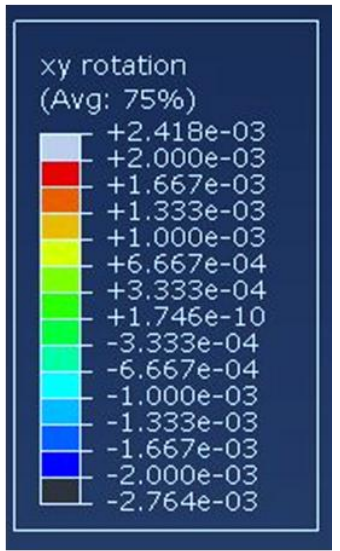

Figure 10. Field plots of EBSD measured (a-b) and CPFE predicted (c-f) in-plane lattice rotation in grain 1 and 2 (first row), and 3 and 4 (second row). Pre-and post-reference-shifted results are shown in (c-d) and (e-f), respectively. The color scale on the left relates to the experimental measurements and that on the right relate to the CPFE prediction. Grain boundaries are marked by the dashed lines.

In order to give a quantitative assessment of the experimentally determined and the CPFE predicted results, all components of the referenced-shifted CPFE results and EBSD measurements along lines A, B, and C introduced in Fig. 6 were extracted and the comparisons are shown in Fig. 11. The referencing clearly accurately reproduces the EBSD observation along line A in a quantitative sense, as can be seen in the first column in Fig. 10. There is good agreement along line B, but the CPFE yy strains (Fig. 11 (e)) are overestimated. Variations in predicted results and EBSD measurements can be seen along line $\mathrm{C}$. 


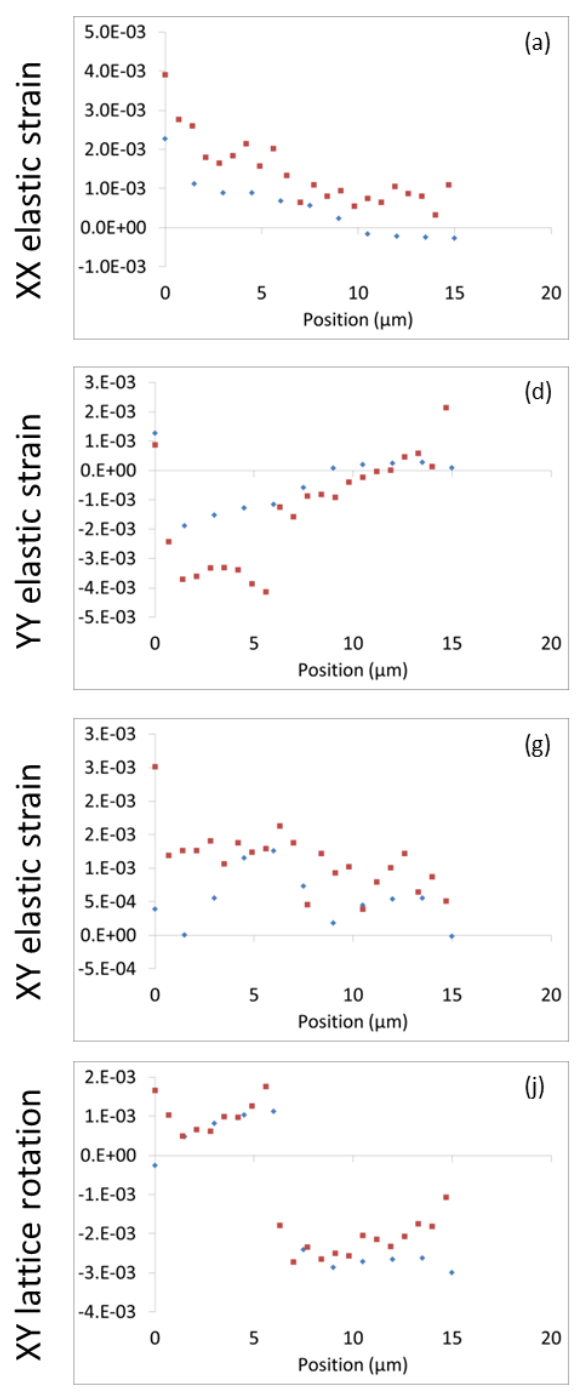

Line A

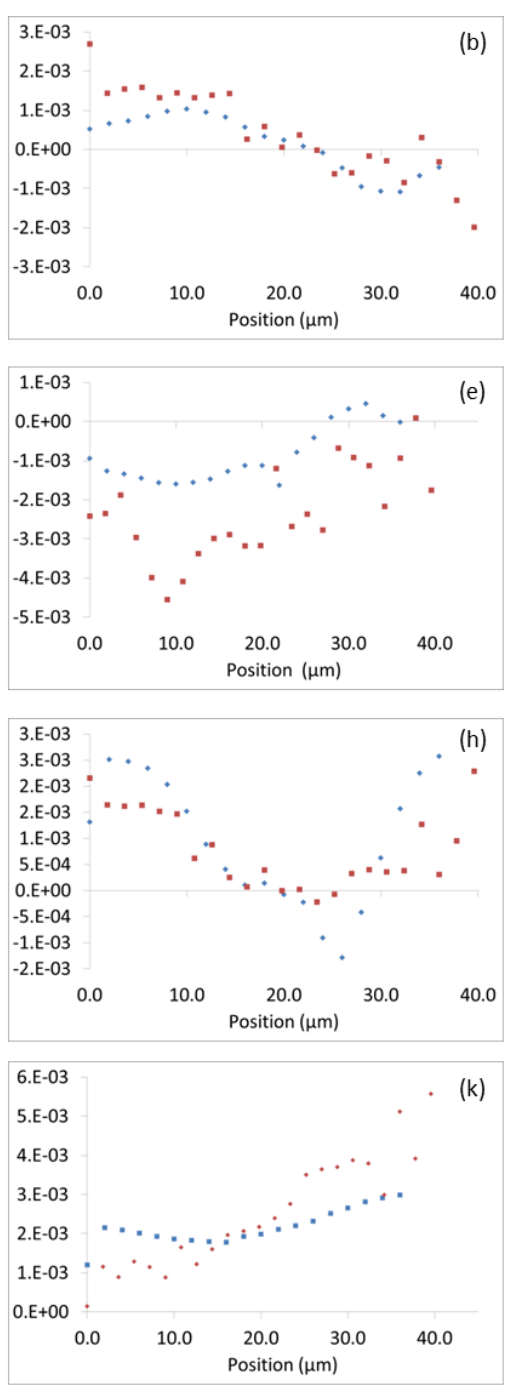

Line B

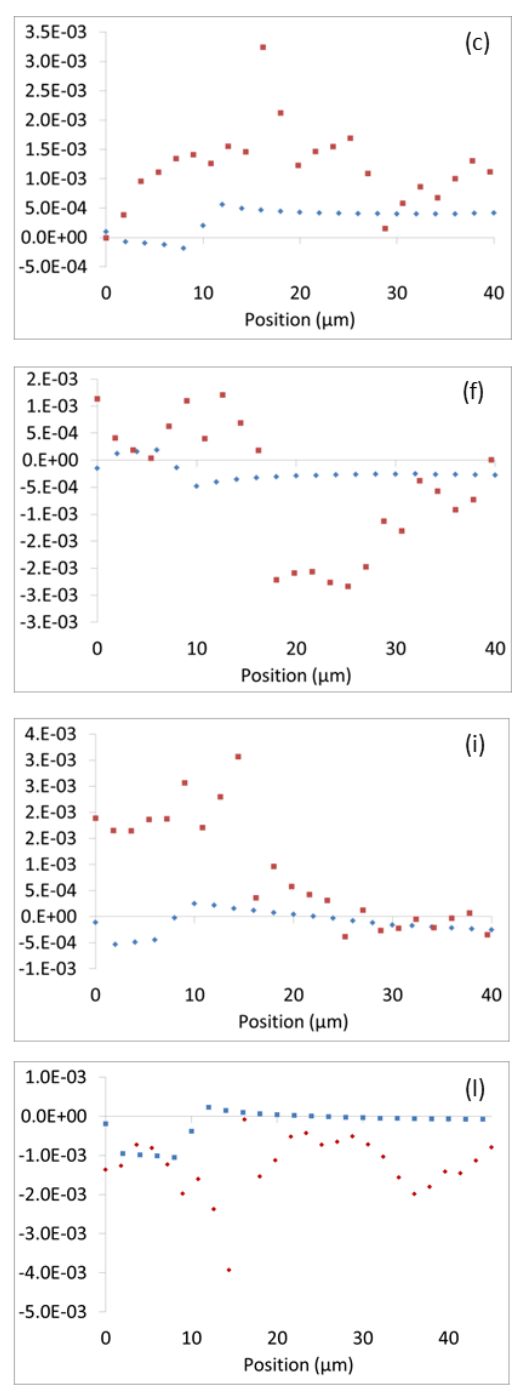

Line C

Figure 11. Reference-shifted CPFE (blue dots) and experimentally determined (red dots) elastic xx

(a-c), yy (d-f), xy (g-i) strains and xy lattice rotation $(j-1)$ for the inclusion/nickel combination subjected to thermal loading. The values are extracted along lines A, B, and C in Fig. 5.

Given the temperature drop, the mismatch in the thermal expansivities of the nickel matrix and the oxides is large enough to generate plasticity local to the inclusion, as the crystal plasticity model predicts in Fig. 12. The CPFE model is employed to predict the local accumulated plastic strains which are developed at the interface because of the thermal mismatch. Accumulated plastic strains [24] of up to about $1 \%$ are observed in the prediction. Therefore, dislocation pile-up at the boundary is anticipated in order to accommodate the large plastic gradients. Indeed, the EBSD measurement 
in Fig. 13 shows GND accumulation at the interface with densities of up to approximately $10^{14}$ $m^{-2}$. GND density is intimately related to lattice curvature which is supported by the Burger's circuit with net Burger's vector. Any dislocation feature that can annihilate the net Burger's vector, such as a dipole, can reduce the number GNDs recovered from EBSD lattice curvature measurement and such annihilated dislocations will be measured as statistically stored dislocations. Dislocation dipoles separated by smaller Burger's vectors (smaller step size) hence potentially yield higher GND densities [42], and this needs to be kept in mind when interpreting GND density.
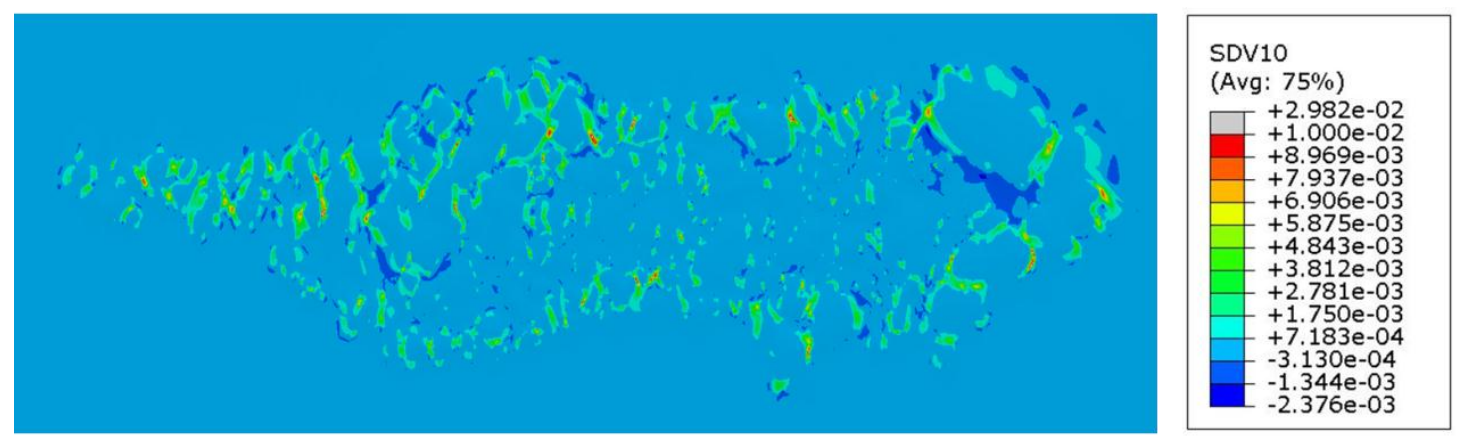

Figure 12. CPFE calculated accumulated plastic strains developed under thermal loading. Highly localized plasticity in the Ni matrix local to the inclusion is seen.
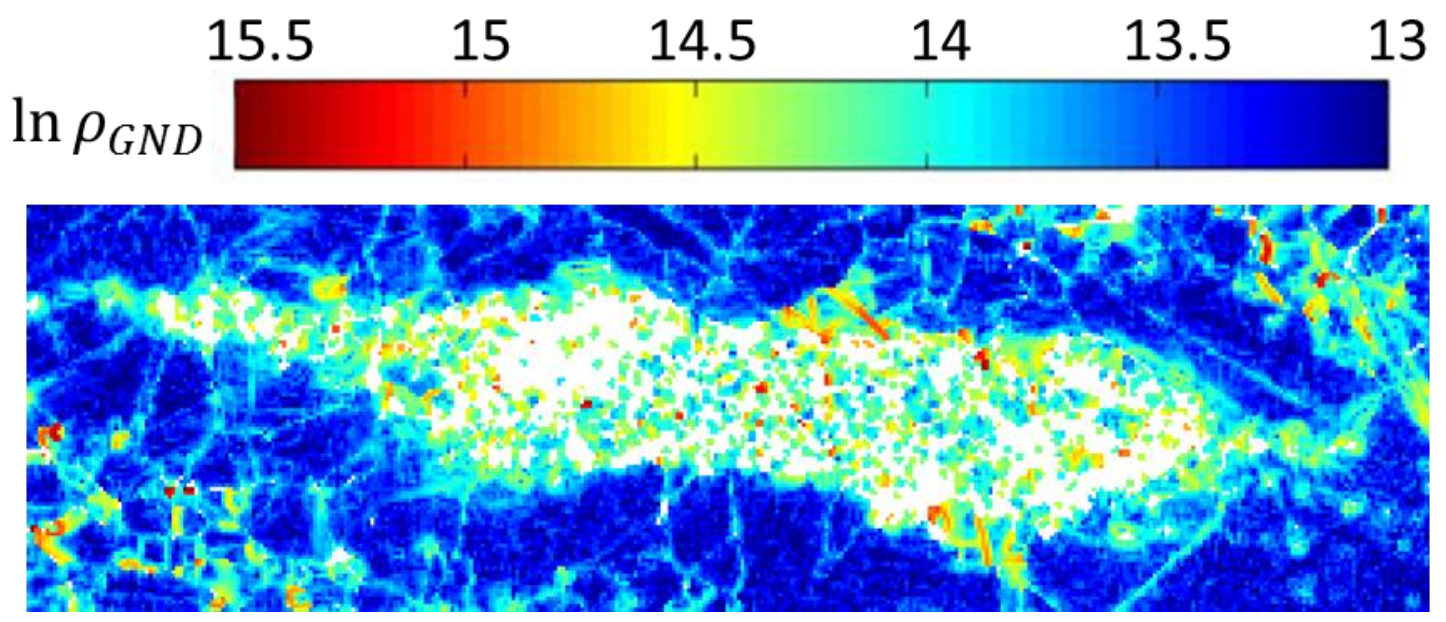

Figure 13. Field plot of experimentally determined GNDs around the inclusion 


\section{Discussion}

In this study, a complex, multi-component non-metallic inclusion embedded in a polycrystalline nickel matrix has been characterized and explicitly modelled. The gradient-enhanced crystal plasticity framework in conjunction with high resolution EBSD has shown the ability to capture the elastic and plastic response under thermal loading in a nickel polycrystal. However, highly localized deformation occurs at the inclusion/matrix interface. This makes the choice of a reference with zero strain or known strain state problematic for the HR-EBSD measurements, since each grain requires a known point of strain in order for referencing. The proposed method, which involves reference shifting within the selected grains, has allowed detailed like-with-like comparisons with the experimental polycrystal measurements to be made. Reasonably good qualitative and quantitative agreement is achieved, enabling us not only to understand the thermal deformation, but also to quantify the residual strains and rotations local to the complex agglomerate prior to any external (e.g. cyclic mechanical and thermal, in-service) loading. This is crucial for further investigation of fatigue behavior of these alloys produced via the same route particularly in the context of crack nucleation. This fundamental study has also provided a potential methodology to overcome the socalled 'reference pattern' problem in EBSD, particularly associated with strain measurement in polycrystal systems. Elastic strains and lattice rotations at a reference point can be predicted by the CPFE model and the determination of absolute values of strains and rotations within the grain can therefore be achieved.

The strain-shifting technique presented is to address just one major issue; that in the HR-EBSD technique, the elastic strains are known but only up to the extent that prior knowledge of the full elastic strain at any one arbitrary point within each grain must be known a priori. Our methodology is to argue that the corresponding elastic strain at each grain's 'reference' point may be estimated using CPFE and hence used to calculate the absolute (as opposed just to relative) EBSD-measured full-field elastic strains. The origin of the elastic strain (e.g. uniform lattice distortion, lattice 
curvature, e.g. through GNDs and support of plastic strain gradients, or both through the establishment of dislocation structure) is not easily determinable but importantly, is not needed to carry out the elastic strain shifting. HR-EBSD captures the elastic strains resulting from the mechanisms listed (but cannot capture, for example, lattice slip or volumetric dilatation); the shifting of these elastic strains is merely a linear operation which doesn't in its own right change the material or stress state, and is only necessary because of the absence of a datum point (e.g. a zero elastic strain point in a given grain). Clearly, total strains and plastic strains cannot be shifted in this way because they relate to nonlinear deformation processes.

A further important point relates to the initial and residual strains. The EBSD measurements are, of course, elastic strains. In the problem addressed, the nickel alloy has been annealed at $1033 \mathrm{~K}$ for sixteen hours prior to slow cooling. The nickel matrix is isotropic in its thermal expansivity and it is argued that because of the long anneal, it is reasonably residual stress-free except at the interfaces of the Ni matrix and agglomerate inclusions because the latter have a different thermal expansivity to that of the nickel. This has been demonstrated experimentally [21] for single crystal Ni containing carbide particles where away from the carbide interfaces, near-zero elastic straining was measured. Hence, upon cooling after the anneal of our agglomerate-containing polycrystal, it is anticipated that there will be residual elastic strains at the locations of the agglomerate $-\mathrm{Ni}$ interfaces, and because HR-EBSD is carried out on cooling, in the absence of any other loading, it is precisely these residual elastic strains which are measured by the HR-EBSD and which are modelled in the CPFE. Indeed, other detailed modelling studies [22] give some confidence in its ability to capture the local elastic residual strains.

While not addressed explicitly in the current paper, the important question arises of what happens if subsequent loading (be it mechanical or thermal or both) is imposed. In connection firstly with EBSD studies, subsequent loading likely leads to early onset of slip (because of the pre-existing 
residual stress state), and change in material state through establishment of dislocation structure. On termination of the subsequent loading, however, an elastic strain state will exist which may be measured (subject to the now well-discussed need to establish a reference strain state) by HR-EBSD. Again, the mechanistic processes leading to this elastic strain state are not needed for its measurement. In addition, CPFE modelling is, in principle, now able to utilise the initial elastic strain state, and corresponding residual stress state, in the modelling of the subsequent loading and is able to take full account of the effect of the initial (or residual) strain state on the initiation of subsequent slip and deformation.

Some of the observed disagreements between the CPFE predictions and the EBSD measurements can be explained on the basis of the assumptions made. First, the oxide particles and nickel grains are assumed to be prismatic through the depth of the model. Although this is likely to be realistic for the large particles and large nickel grains, it is unlikely to be the case for the small grains. A cross-section (Fig. 14) taken from the Al-rich region of the sample indicates that small grains only extend a few microns through the depth, whereas the grains in the inclusion vicinity are several times bigger in size. Sub-surface structure has been found to significantly affect the strain distribution on the free surface [43]. Second, due to lack of inclusion geometry in the depth direction, inter-particle influence cannot be completely eliminated. In fact, particles with a wide range of sizes below the free surface are observed in Fig. 14. Third, a single 3D element layer through the material depth is not sufficient for accurate discretization, leading to over or underpredicted displacements, which, in return, influence quantities on the free surface. Fourth, the presence of other alloying and refractory elements as a result of the processing is of great importance in material properties. 


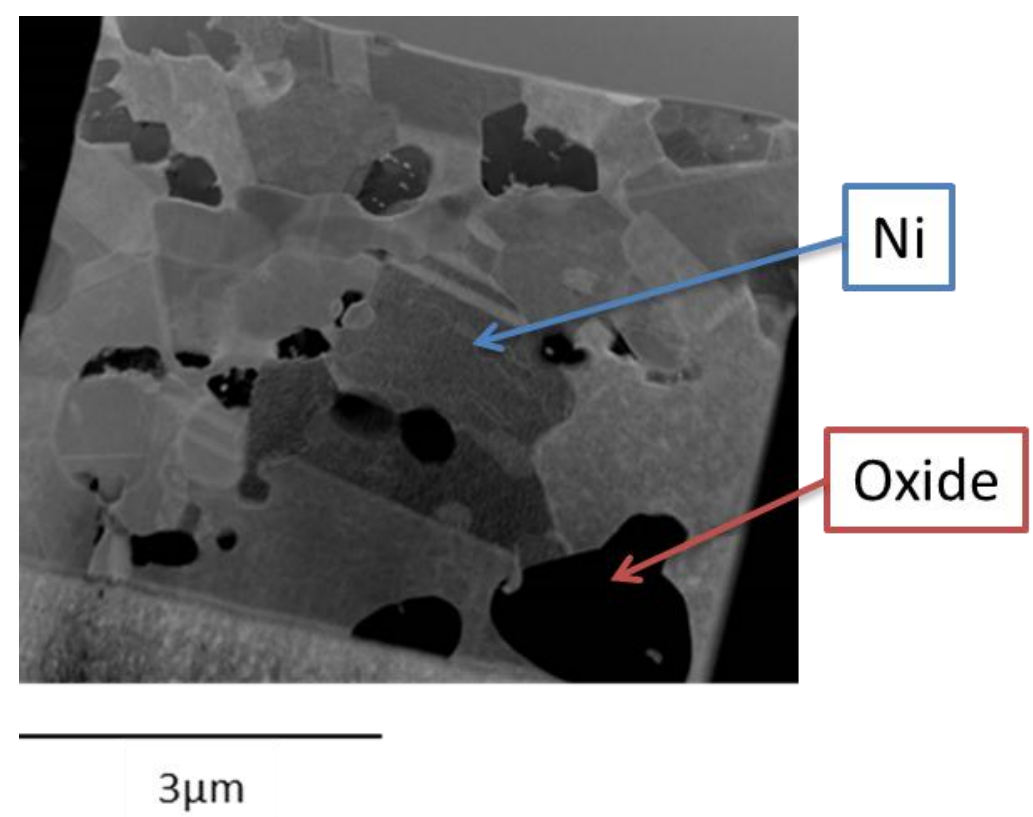

Figure 14. Dark-field TEM image of a cross-section taken from the Al-rich region in the inclusion sample showing nickel grains and oxide particles of various sizes. The free surface is at the top and re-deposition of FIB is seen at the bottom.

GND density is calculated directly by taking derivatives of the lattice rotations. However, a complete determination of GND density requires knowledge of the area over which it is calculated. The step size used in the EBSD measurement is $0.45 \mu \mathrm{m}$, yielding dislocation densities of approximately $10^{14} \mathrm{~m}^{-2}$ at the particle/matrix interface. However, determining the appropriate step size for GND calculation in HR EBSD is difficult. The selected step size should be the same size as the dislocation structures of interest to ensure separation of annihilating opposing dislocation features, such as dipoles, and hence yield appropriate GND measurements [42]. Comparatively, there are numerous studies of dislocation development due to differing thermal expansivities. A qualitative study of dislocation distribution using TEM in Rene 95 has shown dislocation pile-ups near doped alumina particles [45]. Karameched et al has reported GND densities of $5 \times 10^{14} \mathrm{~m}^{-2}$ near a carbide particle in a directionally solidified nickel superalloy [21]. A numerical study on metal matrix composites under thermal-mechanical loading has found GND accumulation with magnitudes of up to $10^{14} \mathrm{~m}^{-2}$ at the inclusion/matrix interface [46]. 


\section{Conclusions}

A PM polycrystalline nickel superalloy sample containing a complex agglomerate of small $\mathrm{Ni}$ grains and oxide particles has been characterized using SEM back-scattered electron imaging, energy-dispersive x-ray, and high-resolution EBSD techniques. The thermal treatment history and differences in thermal expansivities led to the establishment of residual elastic strain fields, lattice rotations and curvatures local to the polycrystal-agglomerate interface which have been quantified using HR-EBSD. A full and explicit representation of the polycrystal Ni and agglomerate has been incorporated within a crystal plasticity finite element model and direct grain-by-grain comparisons of strain and lattice rotation made with the HR-EBSD measurements. Good quantitative and qualitative agreement is obtained.

A remaining draw-back of the HR-EBSD technique for full-field strain mapping is that strains can only be obtained with reference to some known strain value at a designated reference point within (e.g.) a specific grain. Hence, all strains within a grain are given with respect to the grain reference point strain and therefore, comparisons of strain values across grains in a polycrystal are not normally possible using EBSD. However, a methodology for reference shifting using the combined approaches of HR-EBSD and CPFE modelling has been presented such that like-for-like strain comparisons across grains and hence full-field strain mapping is achieved. This approach has allowed the quantification of residual elastic strain fields and the accumulated slip resulting from the presence on a complex non-metallic inclusion in the PM superalloy RR1000.

\section{Acknowledgments}

Tiantian Zhang is grateful for the financial support and supply of nickel superalloy from RollsRoyce plc. The authors much appreciate helpful discussions with Drs Jun Jiang, and Ben Britton (Imperial College), Professor Angus Wilkinson (University of Oxford), and Dr Mark Hardy and Iain Parr (Rolls-Royce plc.). 


\section{Appendix A. Validation of the shifting methodology}

Consider the 2-D structure with multiple grains as shown in Fig. 5 (a) under uniaxial loading $\mathrm{P}$ in the y-direction. Suppose the strain profile in grain $\beta$ is as depicted in Fig. 5 (b) where EBSDmeasured strains within the grain to be shifted (by $\left.\Delta \varepsilon^{0}\right)$ to ensure that the EBSD-measured $\left(\varepsilon^{e \prime}\right)$ and CPFE-calculated $\left(\varepsilon^{e}\right)$ elastic strains at the reference such that for linearity, the relationship between them can be expressed as:

$\varepsilon^{e \prime}=\varepsilon^{e}+\Delta \varepsilon^{0}$

Since the strains measured using HR-EBSD are purely elastic, the stress corresponding to the shifted elastic strain may be determined by

$\boldsymbol{\sigma}^{\prime}=\boldsymbol{D} \boldsymbol{\varepsilon}^{e^{\prime}}=\boldsymbol{D}\left(\boldsymbol{\varepsilon}^{e}+\Delta \boldsymbol{\varepsilon}^{0}\right)=\boldsymbol{D} \boldsymbol{\varepsilon}^{e}+\boldsymbol{D} \Delta \boldsymbol{\varepsilon}^{0}$

where $\boldsymbol{\sigma}^{\prime}$ and $\boldsymbol{\sigma}$ are the shifted and actual stresses respectively, and $\boldsymbol{D}$ is the elastic stiffness matrix.

Also, for the actual stress,

$\boldsymbol{\sigma}=\boldsymbol{D} \boldsymbol{\varepsilon}^{e}$

so that

$\boldsymbol{\sigma}^{\prime}=\boldsymbol{\sigma}+\boldsymbol{D} \Delta \boldsymbol{\varepsilon}^{0}=\boldsymbol{\sigma}+\Delta \boldsymbol{\sigma}^{0}$

where

$\Delta \boldsymbol{\sigma}^{0}=\Delta \boldsymbol{D} \boldsymbol{\varepsilon}^{0}$

\section{Pointwise equilibrium}

To satisfy conditions of static equilibrium, the surface traction exerted on a surface of an arbitrary volume must be zero. In the absence of body forces, the requirement for equilibrium may be written $\nabla \cdot \boldsymbol{\sigma}=\left[\begin{array}{ccc}\frac{\partial}{\partial x} & 0 & \frac{\partial}{\partial y} \\ 0 & \frac{\partial}{\partial y} & \frac{\partial}{\partial x}\end{array}\right]\left[\begin{array}{c}\sigma_{x} \\ \sigma_{y} \\ \sigma_{x y}\end{array}\right]=\left(\begin{array}{l}0 \\ 0 \\ 0\end{array}\right)$.

This requirement may be imposed for both the actual and shifted stresses independently such that 
$\nabla \cdot \boldsymbol{\sigma}^{\prime}=\nabla \cdot\left(\boldsymbol{\sigma}+\Delta \boldsymbol{\sigma}^{0}\right)=\nabla \cdot \boldsymbol{\sigma}+\nabla \cdot \Delta \boldsymbol{\sigma}^{0}$

Because $\Delta \boldsymbol{\sigma}^{0}$ is constant and uniform over a given grain, so

$\nabla \cdot \boldsymbol{\sigma}^{\prime}=\nabla \cdot \boldsymbol{\sigma}$

and hence for satisfaction of pointwise equilibrium,

$\nabla \cdot \sigma=0$

so that from above, if $\boldsymbol{\sigma}$ satisfies equilibrium, then so does $\boldsymbol{\sigma}^{\prime}$.

\section{Overall equilibrium}

Consider the $\mathrm{y}$-direction internal force along line $\mathrm{A}$ through grains $\alpha, \beta$, and $\gamma$, which here $\mathrm{e}$ consider, for simplicity, to have unit depth. The summation of internal forces over the grains must satisfy:

$$
\begin{aligned}
& \int_{\text {Line } A} \sigma_{y y} d x=\int_{\alpha} \sigma_{y y} d x+\int_{\beta} \sigma_{y y} d x+\int_{\gamma} \sigma_{y y} d x \equiv P \\
& =\int_{\alpha}\left(\sigma_{y y}^{\prime}+\Delta \sigma_{y y}^{0, \alpha}\right) d x+\int_{\beta}\left(\sigma_{y y}^{\prime}+\Delta \sigma_{y y}^{0, \beta}\right) d x+\int_{\gamma}\left(\sigma_{y y}^{\prime}+\Delta \sigma_{y y}^{0, \gamma}\right) d x \\
& =\int_{\text {Line A }} \sigma_{y y}^{\prime} d x+\int_{\alpha} \Delta \sigma_{y y}^{0, \alpha} d x+\int_{\beta} \Delta \sigma_{y y}^{0, \beta} d x+\int_{\gamma} \Delta \sigma_{y y}^{0, \gamma} d x
\end{aligned}
$$

Because $\Delta \boldsymbol{\sigma}^{0}$ is constant within each grain irrespective of location, this simplifies to

$$
\int_{\text {Line } A} \sigma_{y y}^{\prime} d x=P-\Delta \sigma_{y y}^{0, \alpha} \int_{\alpha} d x-\Delta \sigma_{y y}^{0, \beta} \int_{\beta} d x-\Delta \sigma_{y y}^{0, \gamma} \int_{\gamma} d x
$$

or equivalently,

$P^{\prime}=P-P^{\alpha}-P^{\beta}-P^{\gamma}$.

Hence the elastic strain shifting corresponds simply to the introduction of body forces, $P^{\alpha}, P^{\beta}$, and $P^{\gamma}$, associated with grains $\alpha, \beta$, and $\gamma$ which are constant and related to the local stress shift, $\Delta \boldsymbol{\sigma}^{0}$, which demonstrates the linearity of the problem.

\section{Compatibility of strains}

Finally, compatibility relations in 2-D require that 
$\frac{\partial^{2} \varepsilon_{x x}}{\partial y^{2}}+\frac{\partial^{2} \varepsilon_{y y}}{\partial x^{2}}=2 \frac{\partial^{2} \varepsilon_{x y}}{\partial x \partial y}$

From above,

$\varepsilon_{x x}^{\prime}=\varepsilon_{x x}+\Delta \varepsilon_{x x}^{0}$

$\varepsilon_{y y}^{\prime}=\varepsilon_{y y}+\Delta \varepsilon_{y y}^{0}$

$\varepsilon_{x y}^{\prime}=\varepsilon_{x y}+\Delta \varepsilon_{x y}^{0}$.

Hence the requirement for compatibility becomes

$\frac{\partial^{2}\left(\varepsilon_{x x}+\Delta \varepsilon_{x x}^{0}\right)}{\partial y^{2}}+\frac{\partial^{2}\left(\varepsilon_{y y}+\Delta \varepsilon_{y y}^{0}\right)}{\partial x^{2}}=2 \frac{\partial^{2}\left(\varepsilon_{x y}+\Delta \varepsilon_{x y}^{0}\right)}{\partial x \partial y}$

which reduces to

$\frac{\partial^{2} \varepsilon_{x x}^{\prime}}{\partial y^{2}}+\frac{\partial^{2} \varepsilon_{y y}^{\prime}}{\partial x^{2}}=2 \frac{\partial^{2} \varepsilon_{x y}^{\prime}}{\partial x \partial y}$

since $\Delta \varepsilon_{x x}^{0}, \Delta \varepsilon_{y y}^{0}$, and $\Delta \varepsilon_{x y}^{0}$ are simply constant terms.

Hence if $\boldsymbol{\varepsilon}$ satisfies compatibility, so does $\boldsymbol{\varepsilon}^{\prime}$.

\section{References}

[1] Kantzos P, Bonacuse P, Telesman J, Gabb T, Barrie R, Banik A. Effect of powder cleanliness on the fatigue behavior of powder metallurgy Ni-disk alloy Udimet 720. In: Green KA, Pollock TM, Harada H, Howson TE, Reed RC, Schirra JJ, Walston S, editors. Superalloys 2004 (Tenth International Symposium), 2004. p.409.

[2] Pang HT, Reed PAS. International Journal of Fatigue 2008;30:2009.

[3] Gabb TP, Bonacuse PJ, Ghosn LJ, Sweeney JW, Chatterjee A, Green KA. Assessments of low cycle fatigue behavior of powder metallurgy alloy U720. In: Center NGR, editor, vol. 1389, 2000. p.110.

[4] Huron ES, Roth PG. The influence of inclusions on low cycle fatigue life in a P/M nickelbase disk superalloy, 1996.

[5] Hyzak JM, Bernstein IM. Metallurgical Transactions a-Physical Metallurgy and Materials Science 1982;13:45.

[6] Alexandre F, Deyber S, Pineau A. Scr. Mater. 2004;50:25.

[7] Findley KO, Saxena A. Metall. Mater. Trans. A-Phys. Metall. Mater. Sci. 2006;37A:1469.

[8] Caton MJ, Jha SK, Rosenberger AH, Larsen JM. Superalloys 2004 (Tenth International Symposium) 2004:305.

[9] Hyzak JM, Bernstein IM. Metallurgical Transactions a-Physical Metallurgy and Materials Science 1982;13:33.

[10] Gabb TP, Telesman J, Kantzos PT, Bonacuse PJ, Barrie RL. Initial assessment of the effects of non-metallic inclusions on fatigue life of powder metallurgy processed Udimet $(R)$ alloy 720. Warrendale: Minerals, Metals \& Materials Soc, 2003.

[11] Wilkinson AJ, Meaden G, Dingley DJ. Ultramicroscopy 2006;106:307.

[12] Wilkinson AJ, Meaden G, Dingley DJ. Materials Science and Technology 2006;22:1271. 
[13] Nye J. Acta metallurgica 1953;1:153.

[14] Britton TB, Liang H, Dunne FPE, Wilkinson AJ. Proc. R. Soc. A-Math. Phys. Eng. Sci. 2010;466:695.

[15] Littlewood PD, Britton TB, Wilkinson AJ. Acta Mater. 2011;59:6489.

[16] Britton TB, Birosca S, Preuss M, Wilkinson AJ. Scr. Mater. 2010;62:639.

[17] Jiang J, Britton TB, Wilkinson AJ. Acta Mater. 2013;61:7227.

[18] Jiang J, Britton TB, Wilkinson AJ. Acta Mater. 2013;61:5895.

[19] Jiang J, Britton TB, Wilkinson AJ. Philosophical Magazine Letters 2012;92:580.

[20] Miyamoto G, Shibata A, Maki T, Furuhara T. Acta Mater. 2009;57:1120.

[21] Karamched PS, Wilkinson AJ. Acta Mater. 2011;59:263.

[22] Dunne FPE, Kiwanuka R, Wilkinson AJ. Proc. R. Soc. A-Math. Phys. Eng. Sci. 2012;468:2509.

[23] Wilkinson AJ, Britton TB. Materials Today 2012;15:366.

[24] Dunne FPE, Rugg D, Walker A. Int. J. Plast. 2007;23:1061.

[25] McDowell DL, Dunne FPE. International Journal of Fatigue 2010;32:1521.

[26] Dunne FPE, Rugg D. Fatigue Fract. Eng. Mater. Struct. 2008;31:949.

[27] Sweeney CA, Vorster W, Leen SB, Sakurada E, McHugh PE, Dunne FPE. J. Mech. Phys. Solids 2013;61:1224.

[28] Rugg D, Britton TB, Gong J, Wilkinson AJ, Bagot PAJ. Materials Science and Engineering: A 2014;599:166.

[29] Kacher J, Landon C, Adams BL, Fullwood D. Ultramicroscopy 2009;109:1148.

[30] Britton TB, Maurice C, Fortunier R, Driver JH, Day AP, Meaden G, Dingley DJ, Mingard K, Wilkinson AJ. Ultramicroscopy 2010;110:1443.

[31] J.W.Christian. The Theory of Transformations in Metals and Alloys: Pergamon, 2002.

[32] Hessel SJ, Voice W, James AW, Blackham SA, Small CJ, Winstone MR. Nickel alloy for turbine engine component. Google Patents, 1997.

[33] Jablonski DA. Materials Science and Engineering 1981;48:189.

[34] Shamblen CE, Chang DR. Metallurgical Transactions B-Process Metallurgy 1985;16:775.

[35] Findley KO, Evans JL, Saxena A. International Materials Reviews 2011;56:49.

[36] Wilkinson AJ, Clarke EE, Britton TB, Littlewood P, Karamched PS. J. Strain Anal. Eng. Des. 2010;45:365.

[37] Wilkinson AJ, Randman D. Philosophical Magazine 2010;90:1159.

[38] Britton TB, Wilkinson AJ. Ultramicroscopy 2012;114:82.

[39] Lee EH. Journal of Applied Mechanics 1969;36:1.

[40] Wang J, Li HP, Stevens R. J Mater Sci 1992;27:5397.

[41] Prasannavenkatesan R, Przybyla CP, Salajegheh N, McDowell DL. Engineering Fracture Mechanics 2011;78:1140.

[42] Jiang J, Britton TB, Wilkinson AJ. Ultramicroscopy 2013;125:1.

[43] Kartal ME, Dunne FPE, Wilkinson AJ. Acta Mater. 2012;60:5300.

[44] Britton TB, Wilkinson AJ. Ultramicroscopy 2011;111:1395.

[45] Xie X, Zhang L, Zhang M, Dong J, Bain K. Superalloys, TMS 2004:451.

[46] Aghababaei R, Joshi SP. Int. J. Plast. 2013;42:65. 